\title{
Phantomgrenzen als heuristisches Konzept für die Grenzforschung
}

\author{
Béatrice von Hirschhausen
}

\begin{abstract}
Dieser Beitrag definiert das Konzept Phantomgrenzen und erläutert sein heuristisches Potenzial in der Regionalforschung. Phantomgrenzen bezeichnen die Spuren vergangener territorialer Ordnungen, welche in aktuellen Räumen feststellbar sind. Dabei wird insbesondere auf die Wechselwirkungen zwischen drei nach Henri Lefebvre (1974) identifizierbaren Ebenen der Raumproduktion geachtet: Raumerfahrung, Raumimagination sowie Raumgestaltung. Die Identifikation und Interpretation von Phantomgrenzen ist kontextspezifisch und ermöglicht nicht nur einen neuen Blick auf die Entwicklung Ostmitteleuropas - der Region, in der die Forschung zu Phantomgrenzen erstmalig angewendet wurde -, sondern auch auf andere geografisch-historische Räume.
\end{abstract}

\section{Schlagwörter}

Phantomgrenzen, Phantomräume, Regionalforschung, Ostmitteleuropa

\section{Einleitung}

Dieser Beitrag behandelt das Konzept der Phantomgrenzen bzw. der von ihnen implizierten Phantomräume, sowohl konzeptionell als auch anhand ausgewählter Fallstudien. Ein wesentlicher Motor bei der Entwicklung des Konzepts der Phantomgrenzen war die breit rezipierte Kontroverse bezüglich einer spezifischen historischen Region: Südosteuropa. Dabei wird die ,Realität' von durch Historiker umrissene geografische Regionen in Frage gestellt, welche im Folgenden als Geschichtsregionen bezeichnet werden.

Die Auseinandersetzung um Geschichtsregionen (vgl. Troebst 2010) und deren Merkmale und Grenzen wurde in den 1990er-Jahren für die Region Südosteuropa maßgeblich zwischen dem Berliner Historiker Holm Sundhaussen und der bulgarisch-amerikanischen Historikerin Maria Todorova ausgetragen. Die Diskussion spiegelte die Kontroverse zweier Pole in der Regionalforschung wider:

- Todorova (1997) hatte bei ihrer Dekonstruktion der Kategorie ,Balkan` als Nebenprodukt eines abendländischen stigmatisierenden Diskurses über ,Balkanismus‘ die Realität der Geschichtsregion selbst in Frage gestellt;

- Holm Sundhaussen (1999) hatte dagegen einen im Kern strukturalistischen Ansatz gewählt, welcher auf der Sinnhaftigkeit solcher historischen, wenn auch durchlässigen Raumkonstruktionen zum Verständnis der dortigen Gesellschaften beharrte.

Die Kontroverse ging in einem Formelkompromiss auf, der die Geschichtsregionen zwar nicht als naturalisierte regionale Raumformate deutet, aber auch keine weiteren alternativen Ansätze anbietet (vgl. Todorova 2002; Sundhaussen 2003). Damit blieb die Frage nach der zeitlichen Dimension von Räumen unbeantwortet, ebenso wie diejenige nach der Fortwirkung bestimmter regional geprägter kultureller und sozialer Muster im Handeln der Bevölkerung - trotz erheblicher Epochenbrüche und neuen, sich ändernden politischen Grenzziehungen. Diese Fra- 
gen wurden zum Ausgangpunkt der konzeptionellen Überlegungen zu Phantomgrenzen am Beispiel Ostmitteleuropas.

Der folgende Beitrag fasst Ergebnisse des Forschungsprojekts „Phantomgrenzen in Ostmitteleuropa “ zusammen, welches zwischen 2011 und 2017 am Beispiel Ostmittel- und Südosteuropas im Rahmen eines interdisziplinären Verbundprojekts entwickelt wurde. Im Kern dieser Forschung stehen soziale Phänomene, welche sich statistisch erheben lassen und kartografisch darstellbar sind. Sie beschäftigen sich mit Menschen und Akteursgruppen, welche durch ihr konkretes alltägliches Handeln und ihre Entscheidungen solche Phantome, von unten' auf der Karte erscheinen lassen. Das Phantomgrenzen-Konzept und die damit verbundenen Thesen wurden durch einen interdisziplinären und induktiven, von Fallstudien ausgehenden Forschungsansatz geprüft und entwickelt; konkrete empirische Beispiele dienen der Veranschaulichung. ${ }^{1}$

\section{Phantomgrenzen - eine heuristische Annäherung}

Die physische Markierung einer Grenze sowie ihre lokale Wirkung entlang einer Grenzlinie und in ihrer direkten Umgebung können binnen kurzer Zeit verschwinden, ohne Spuren zu hinterlassen: So können Zäune oder Grenzposten mit Schlagbäumen und Grenzkontrollen durch politische Entscheidungen abgeschafft werden bzw. ihre ursprüngliche Bedeutung verlieren. Dies trifft auch für ,festere' Grenzen zu: Ohne Erinnerungspolitik und Musealisierung wären zum Beispiel die Berliner Mauer und die innerdeutsche Grenze des Kalten Krieges schon ein paar Jahre nach ihrer Abschaffung aus der Landschaft verschwunden. Die Räume aber, welche durch Territorialisierungs- sowie Vergesellschaftungsprozesse innerhalb der ehemaligen Grenzen geschaffen wurden, entwickeln sich auf einer wesentlich längeren Zeitachse (vgl. Löwis 2014b; Hirschhausen et al. 2019 mit weiteren Beispielen).

\subsection{Beispiele von Phantomgrenzen in Infrastrukturen und Wahlverhalten}

Betrachten wir Strukturen und Institutionen, die von politischen Akteuren geschaffen worden sind, verändern sich diese mitnichten innerhalb kurzer Zeiträume: Territoriale Gliederungen prägen den Raum langfristig in seiner physischen Gestalt sowie seine Ausstattung mit raumstrukturierenden Funktionseinrichtungen wie Straßen, Gleisen oder Brücken. Ein Blick auf die Karte des Streckennetzes der polnischen Staatsbahn in den 1950er-Jahren (Abb. 1) zum Beispiel lässt die Spuren der früheren imperialen Grenzen erscheinen, die im 19. Jahrhundert das Territorium Polens zwischen dem Russischen Reich, dem preußischen bzw. Deutschen Reich und dem habsburgischen Reich geteilt hatten. 35 Jahre nach der Wiedergründung des polnischen Staates im Jahr 1918 war das Bahnnetz in den zu Preußen gehörenden westlichen

1 Das Kompetenznetzwerk „Phantomgrenzen in Ostmitteleuropa“ wurde von 2011 bis 2017 vom deutschen Bundesministerium für Bildung und Forschung (BMBF) im Rahmen der Förderung der Regionalstudien finanziert. Das Centre Marc Bloch, das Institut für Geschichtswissenschaften der Humboldt-Universität (Südosteuropäische Geschichte), das Zentrum Moderner Orient in Berlin und das Aleksander Brückner Zentrum (Institut für Geschichte der Martin-Luther-Universität Halle-Wittenberg) leiteten jeweils ein Teilprojekt und vernetzten sich darüber hinaus auch mit weiteren Organisationen, darunter das GWZO, das Center B/ORDERS IN MOTION (Europa-Universität Viadrina), das Institut für Länderkunde, das Herder-Institut in Marburg, die Universität Siegen (Europäische Zeitgeschichte nach 1945) und mehrere Forschungsinstitutionen in Polen, der Ukraine, Rumänien, Kroatien und Serbien. Für weitere Informationen siehe die Darstellung des Projektes auf der Webseite www.phantomgrenzen.eu. 
und nördlichen Woiwodschaften (Kreise) wesentlich dichter als in den anderen Regionen des Landes (vgl. Müller 2014).

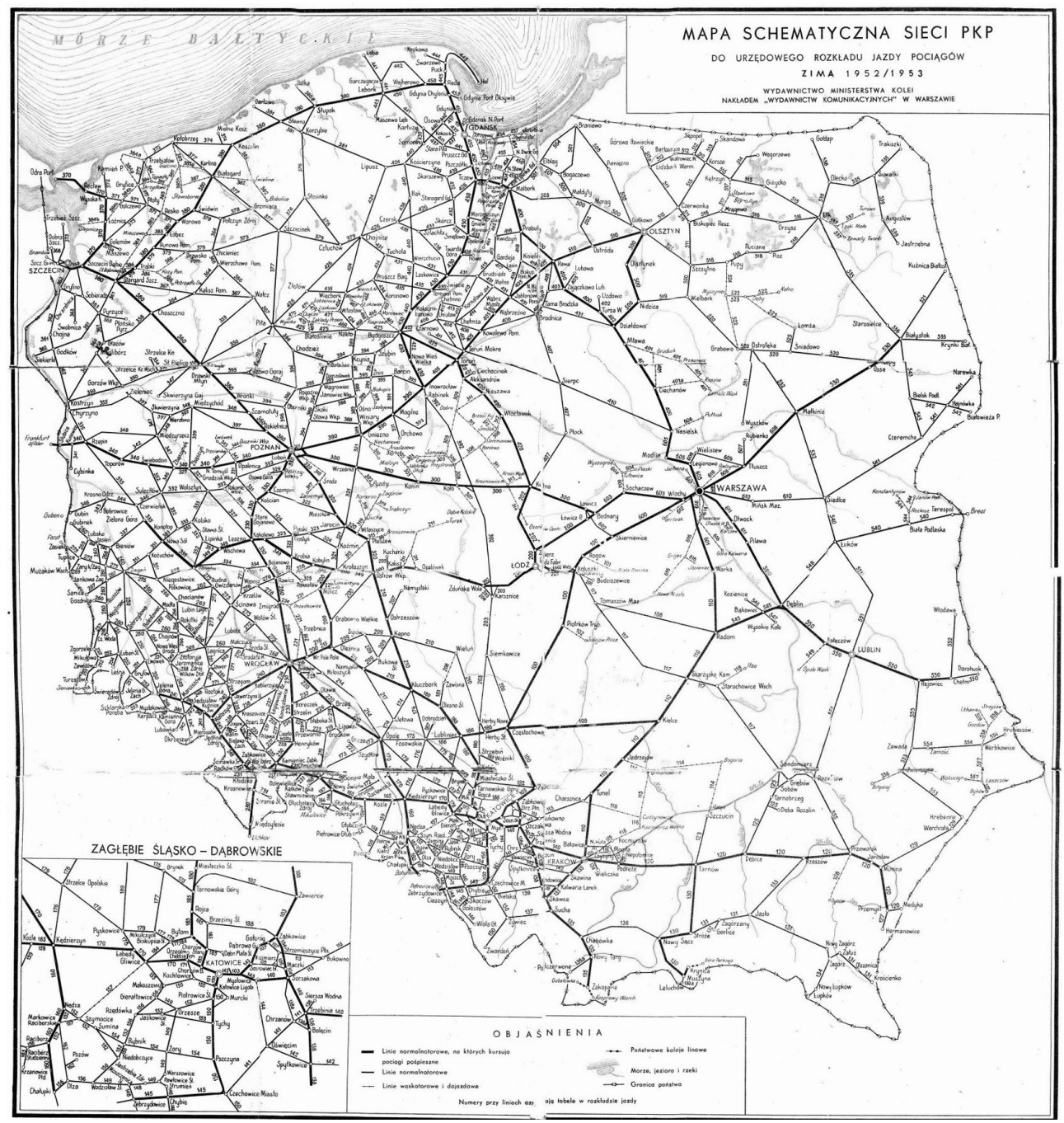

Abb. 1: Eisenbahnnetz der polnischen Staatsbahn PKP in 1952-1953

Quelle: "Mapa Schematyczna Sieci PKP" ZIMA 1952/1953 (https://upload.wikimedia.org/wikipedia/com mons/2/22/PKP1952-53.jp)

Der Aufbau des Eisenbahnnetzes in der zweiten Hälfte des 19. Jahrhunderts hatte den Raum stark und dauerhaft strukturiert. Dabei hatten sowohl die politischen Grenzen dieser Zeit als auch die wirtschaftlichen Asymmetrien zwischen Preußen und den Randgebieten Russlands und Österreich-Ungarns jahrzehntelang überdauert. Die traditionelle Prägung des Raumes lässt sich auch in der Architektur sowie städtischen und dörflichen Siedlungsstrukturen erkennen, welche trotz der neuen Raumgliederung die Landschaften weiter markieren (vgl. Hartshorne 1933). Eine solche Ungleichzeitigkeit lässt sich nicht nur an der materiellen, sondern auch der 
sozialen Prägung des Raumes beobachten. Eine durch bestimmte Agrarpolitik geschaffene Bodenverwaltung kann zum Beispiel die Abschaffung alter Grenzen überdauern. So wurden die Bodenstrukturen der kollektivierten Landwirtschaft der DDR nach der deutschen Wiedervereinigung weitgehend erhalten und in Großbetriebe überführt, welche heute die Landwirtschaft der neuen Bundesländer dominieren. Die von Walter Roubitschek (2004, S. 119) erstellte Karte der „Anteile der Betriebe $>100$ ha“ zeigt die abweichenden Produktionssysteme der neuen Bundesländer zehn Jahre nach der deutschen Wiedervereinigung deutlich auf. Schließlich können auch immaterielle Strukturen die Existenz eines Staates lange überdauern: Dies wird in Fallstudien zur Rechtspflege im Katasterwesen in Rumänien deutlich (vgl. Siegrist/Müller 2015; Müller/Struve 2017, S. 9-11), aber auch in einer früheren Arbeit zu Rechtskulturen und tradierten Normen in Polen (Kraft 2002, S. 75-151).

Besonders erstaunlich sind Spuren vergangener Territorien, die nicht nur in bestimmten formellen und informellen Institutionen als Restformen oder partielle Hinterlassenschaften im Alltagsleben zu erkennen sind, sondern in der kartografischen Visualisierung empirischer Daten, wie z.B. der demografischen Entwicklung, dem Wahlverhalten oder anderen sozialen Praktiken, erscheinen (vgl. Löwis 2015; Hirschhausen et al. 2019). So lassen die Wahlkarten Polens, Rumäniens oder der Ukraine insbesondere bei Präsidentschaftswahlen seit einem Vierteljahrhundert bei einigen Wahlgängen die alten Grenzen der Imperien ,lebendig‘ werden, welche diese Gebiete vor mehreren Generationen unter sich aufgeteilt hatten. Die Stimmenanteile zum zweiten Wahlgang der Präsidentschaftswahl Polens (Abb. 2) zum Beispiel zeigt regional stark divergierende Wahlergebnisse in Ost- und Westpolen, bei denen das Fortwirken sowohl der Grenzziehungen nach dem Ersten Weltkrieg als auch der Grenzen aus der Teilungszeit vom Ende des 18. Jahrhunderts bis 1918 erkennbar sind (vgl. Grosfeld/Zhuravskaya 2014; Zarycki 2015).

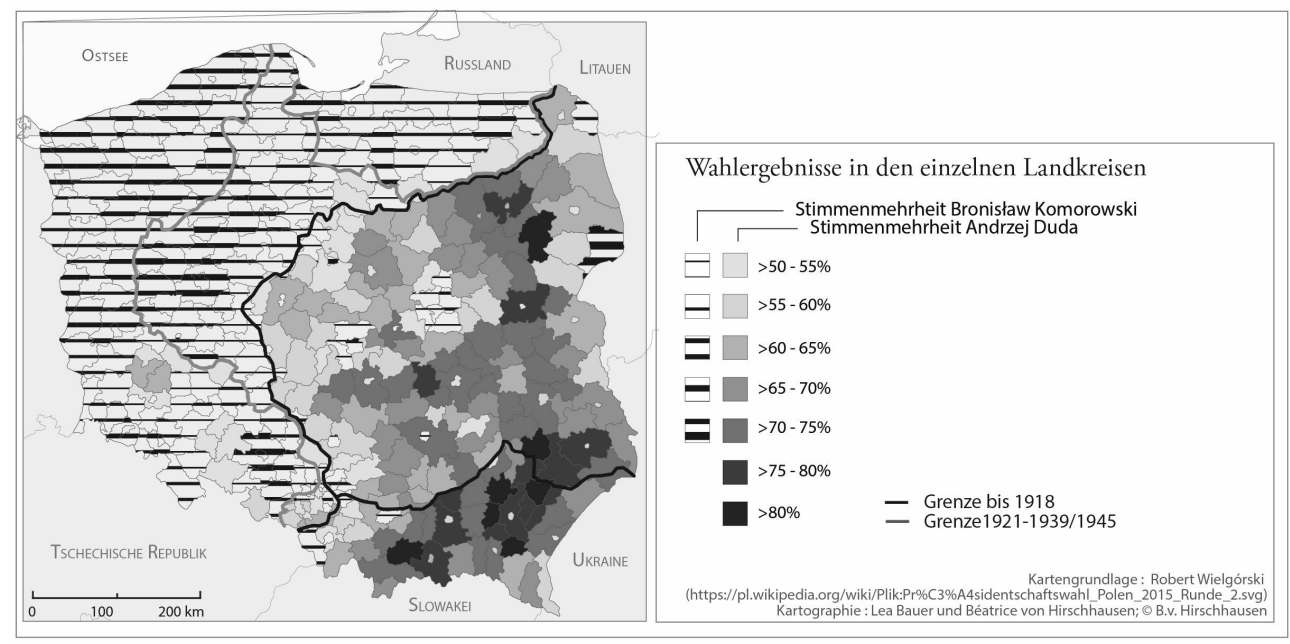

Abb. 2: Stimmenanteile der Kandidaten der zweiten Runde der Präsidentschaftswahl in Polen 2015

Quelle: Esch/Hirschhausen 2017, S. 42 


\subsection{Eine erste Arbeitsdefinition}

Auf einer ersten deskriptiven Ebene bezeichnen Phantomgrenzen die Spuren vergangener territorialer Ordnungen, welche in aktuellen Räumen feststellbar sind (vgl. Grandits et al. 2015, S. 18). Anders als die ,unsichtbaren Grenzen` oder die Grenzziehungen zwischen ethnischen Gruppen (boundaries, vgl. Barth 1969), welche als Begegnungszonen zwischen den Gemeinschaften wirken, ihren Austausch regulieren und stetig hinsichtlich Überschreitbarkeit und Transgression neu verhandelt werden, können sich Phantomgrenzen ohne Grenzverletzung überschreiten lassen. Sie erscheinen weniger als Grenzen denn als Diskontinuitäten: Lokalen Bevölkerungen sind sie oft gar nicht oder nur in diffuser Weise bewusst.

Der Begriff Phantomgrenze ist eine Metapher: Wie sogenannte Phantomschmerzen im doch amputierten Teil eines menschlichen Körpers verspürt werden, machen Phantomgrenzen die Spuren nicht mehr existierender politischer Körperschaften und ihrer Außengrenzen empirisch greifbar. In vielen Fällen wirken historische Räume bzw. die Ergebnisse ihrer Fragmentierung (beispielsweise das Habsburger Reich, das Osmanische Reich, die Teilung Deutschlands oder die Teilungen Polens) fort oder tauchen erneut auf. $\mathrm{Zu}$ Phantomen werden sie aber nicht zuletzt durch ihre Unberechenbarkeit: Die Spuren ,verstorbener' Territorien erscheinen mal flüchtiger, mal über einen längeren Zeitraum hinweg: Zu einem gleichen historischen Zeitpunkt können sie die Kartografie bestimmter Daten prägen, zu anderen jedoch nicht (vgl. Grandits et al. 2015, S. 19).

\section{Phantomgrenzen als Herausforderung für die Forschung}

Weltweit haben staatliche Zerfalls- und Einigungsprozesse immer wieder dazu geführt, dass sich Menschen mit unterschiedlichen Erfahrungen in neuen Zusammenhängen wiedergefunden haben, wobei ihr Alltagshandeln über längere Zeiträume von politischen, wirtschaftlichen, rechtlichen und kulturellen Gepflogenheiten des alten Kontextes beeinflusst wurde. Insbesondere Ostmittel- und Südosteuropa sind Regionen, in denen Prozesse neuer Grenzziehungen in der jüngsten Geschichte ganz besonders oft und besonders eindringlich das politische und gesellschaftliche Leben prägten. Seit dem späten 18. Jahrhundert mussten sich Menschen in diesem Raum wiederholt an veränderte Staatsgrenzen gewöhnen (Abb. 3; Foucher 1993, S. 41-44; Ther 2003; Puttkamer 2010). Hier scheint die politische Landkarte bis in die Gegenwart besonders beweglich und bietet ein komplexes Forschungsfeld. 


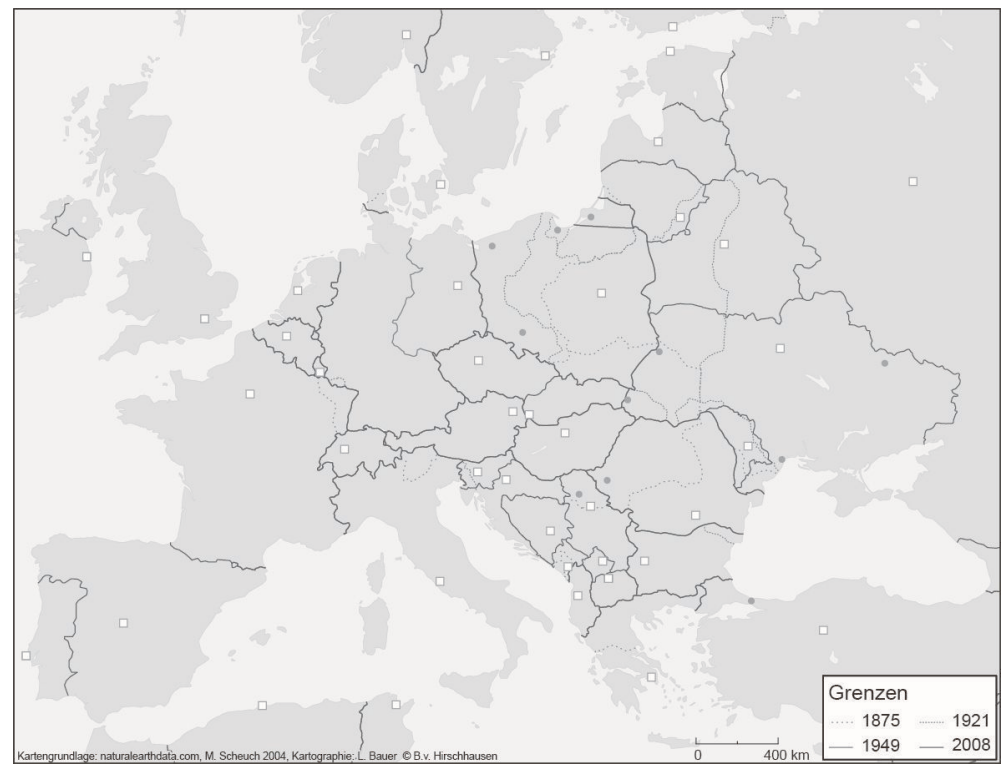

Abb. 3: Palimpsest der Grenzen Ostmittel- und Südosteuropa 1875-2014

Quelle: Esch/Hirschhausen 2017, S. 45.

Phantomgrenzen werden als instabile soziale Phänomene verstanden, welche situativ betrachtet werden sollen. Instabil deshalb, weil sie nicht in Strukturen befestigt, sondern kontextabhängig sind und auf die Handlungen und Wahrnehmungen der Akteure angewiesen sind. Sozial, weil sie nicht rein diskursiv produziert werden, sondern im Alltag und durch Praxis aktualisiert, wiedererfunden oder umgekehrt ignoriert und bis zum Verschwinden vergessen werden können. So können sie weder naturalisiert noch auf rein willkürliche diskursive Kreationen reduziert werden. Besondere Bedeutung kommt dabei den Wechselwirkungen zwischen drei nach Henri Lefebvre (1974) identifizierbaren Ebenen der Raumproduktion zu: des Raumimaginativs, der Raumerfahrung sowie der Raumgestaltung. Eine dezidiert akteurszentrierte Perspektive ermöglicht es, den Bogen zwischen strukturgeprägten Erfahrungen und Imaginativen zu spannen, wie im folgenden Abschnitt ausgeführt wird.

\section{Die drei verschränkten Ebenen der Raumanalyse: Erfahrung - Imaginativ - Gestaltung}

Der Phantomgrenzen-Ansatz sieht vor, Spuren vergangener Grenzen sowie territorialer Ordnungen auf verschiedenen Ebenen zu untersuchen, die aufeinander bezogen sind und sich gegenseitig verstärken oder abschwächen können. Wir gehen davon aus, dass Phantomgrenzen und -räume von Akteuren gleichzeitig auf drei Weisen rezipiert und gleichzeitig geschaffen werden, welche sich gegenseitig beeinflussen: Sie werden 1) erfahren, d.h. von Akteuren und wissenschaftlichen Beobachtern als Erfahrung wahrgenommen, 2) imaginiert, d.h. diskursiv produziert und weitervermittelt, und 3) gestaltet, z.B. durch Territorialisierungsprozesse in der Praxis aktualisiert. Im Zentrum des Phantomgrenzen-Konzeptes stehen damit die Wechselwir- 
kungen zwischen Raumerfahrung, Raumimaginativ ${ }^{2}$ und Raumgestaltung (Esch/Hirschhausen 2017, S. 12-17).

\begin{tabular}{|l|l|l|}
\hline Dimension & Raumform & Ebene der Phantomgrenzen \\
\hline $\begin{array}{l}\text { Raumerfah- } \\
\text { rung }\end{array}$ & vorgefundener Raum & $\begin{array}{l}\text { Phantomgrenzen sind gespeichert in sozialen, men- } \\
\text { talen und räumlichen Strukturen }\end{array}$ \\
\hline $\begin{array}{l}\text { Raumimagina- } \\
\text { tiv }\end{array}$ & bedeutsamer Raum & $\begin{array}{l}\text { Phantomgrenzen werden in Diskursen, Narrativen } \\
\text { und mental maps wahrgenommen und tradiert }\end{array}$ \\
\hline $\begin{array}{l}\text { Raumgestal- } \\
\text { tung }\end{array}$ & praktizierter Raum & $\begin{array}{l}\text { Phantomgrenzen werden durch die Praktiken der } \\
\text { Akteure produziert, reproduziert, aktualisiert aber } \\
\text { auch gelöscht; sie gestalten den Raum mit }\end{array}$ \\
\hline
\end{tabular}

Abb. 4: Die drei verschränkten Dimensionen der Analyse Phantomgrenzen

Quelle: Eigene Darstellung, angelehnt an Esch/Hirschhausen 2017, S. 13.

\subsection{Raumerfahrung}

Die Ebene der Erfahrung wird hier in Anlehnung an Reinhart Koselleck (1984) als gleichzeitig individuell und intersubjektiv begriffen. Auf der Ebene des Individuums handelt es sich um vergegenwärtigte und im Handeln mobilisierbare Vergangenheit, um gelerntes und bewusstes Wissen, aber ebenso um Konventionen, die in Alltagspraktiken übergegangen sind. Auf der intersubjektiven Ebene wird Erfahrung in formellen und informellen Regelsystemen gespeichert, die sich im Laufe der Zeit und über mehrere Generationen hinweg etablieren bzw. allmählich verändern. Diese Erfahrung kann wissentlich vergegenwärtigt werden, Gegenstand einer offiziellen Gedenkpolitik sein, aber auch unbewusst in Habitus, Routine und soziale Morphologie eingehen. In den Worten von Koselleck (1984, S. 354):

„Erfahrung ist gegenwärtige Vergangenheit, deren Ereignisse einverleibt worden sind und erinnert werden können. Sowohl rationale Verarbeitung wie unbewusste Verhaltensweisen, die nicht oder nicht mehr im Wissen präsent sein müssen, schließen sich in der Erfahrung zusammen. Ferner ist in der je eigenen Erfahrung, durch Generationen oder Institutionen vermittelt, immer fremde Erfahrung enthalten und aufgehoben.“

Phantomräume und -grenzen werden sowohl von der Gesellschaft als auch individuell von den Akteuren erfahren, u.a. in gespeichertem und tradiertem Wissen, in dem praktischen Sinn und dem Erlernten, wie es im Habitus, in Normen, Institutionen und sozialer Morphologie oder in der vorgefundenen, quasi materiell kondensierten Raumstruktur niedergelegt ist. Die Raumerfahrung ist dabei teilweise praktisch ,gefangen“ in der Materialität, Gegebenheit und zweifelsfreien Wirksamkeit des vorgefundenen Raumes. Jedoch ist die Erfahrung selbst keine stabile Gegebenheit, sondern eine ständig von den Akteuren neu erfundene und geformte soziale Anordnung der vorgefundenen Gestaltung von Erfahrung. Akteure haben Spielraum bei der

2 Mit „Raumimaginativ“ ist mehr gemeint, als es die Übersetzung von Derek Gregorys (1994) „geographical imaginations“ in „geographische Imaginationen“ oder „Vorstellungen“ ausdrücken würde. Wir haben es nämlich hier mit einem höheren, Wirklichkeitsgehalt‘ zu tun und verwenden daher den im Deutschen seltener gebrauchten Begriff des „Imaginativs“ bzw „Raumimaginativs“, welcher - ähnlich dem französischen imaginaire - auf ein zu einem geografischen Abbild bzw. zu einer Karte geronnenes Narrativ verweist. 
Gestaltung ihres Erfahrungsraums, wie sie ihn begehen, Sinn stiften und ihn verwenden und dadurch wiederum hervorbringen: „vorgefundene Gestaltung bestätigend, ihr ausweichend, sie transformierend, durchbrechend, neu erfindend“ (Esch/Hirschhausen 2017, S. 54).

\section{Beispiel: Raumerfahrung im habsburgischen Erbe}

Das Konzept der Raumerfahrung lässt sich seit den 1980er-Jahren anhand der ,Renaissance des habsburgischen Erbes in den Territorien des ehemaligen Reichs, d.h. den konkreten Nachfolgestaaten, beispielhaft aufzeigen. Zu Zeiten des triumphierenden Sozialismus der 1960erund 1970er -Jahre wurden die institutionellen architektonischen und landschaftlichen Spuren des Reichs von den lokalen Akteuren meistens ignoriert. Diese Spuren fanden sich u.a. im polnischen bzw. ukrainischen Teil von Galizien wieder, auch in der serbischen Vojvodina oder dem rumänischen Banat (vgl. Tomić 2016). Den Akteuren sagten diese Spuren wenig, sie wurden weder von politischen Versuchen der Wiedererstellung von Eigentum noch durch Identitätsdiskurse in Anspruch genommen. Der vorwärtsschauende Blick des sozialistischen Modernisierungsprojekts stellte die historischen Spuren in den Schatten bzw. deklarierte sie sogar als illegitim. So verfiel das barocke Erbe in Ruinen und das Interesse an der österreichisch-ungarischen Doppelmonarchie verkam zu einer Nebenbeschäftigung von Historikern.

Erst im Laufe der 1980er-Jahre wurde dem habsburgischen Erbe wieder ein Sinn zugesprochen und die lokalen Erinnerungen der Angehörigkeit zum vormaligen Imperium wiedererweckt. Hier vermuten wir, dass dieser Trend durch die immer tiefer greifende Enttäuschung des kollektiven Glaubens an den Erfolg des sozialistischen Projekts getrieben war. Des Weiteren spielte auch die sukzessive grenzüberschreitende Diffusion des Konzepts von ,Zentraleuropa' in den intellektuellen Eliten der Region eine wichtige Rolle: Emblematisch dafür ist der berühmte Artikel „Un occident kidanppé ou la tragédie de l'Europe centrale“ von Milan Kundera (1983; 1987; siehe auch Ash 1986). Der Zusammenbruch der sozialistischen Regime hat die regionale Wahrnehmung der Gesellschaft dann noch weitgehend beeinflusst. Dies spiegelt sich in der materiellen Wirklichkeit der Landschaften, der Architektur und des Kulturwerkes wider, welche seit drei Jahrzehnten restauriert werden und somit ihren alten Glanz wiederfinden: Sie erfahren zunehmend breite Erwähnung in der touristischen Literatur und werden in einigen journalistischen Berichten zelebriert. Das habsburgische Erbe wird aber auch von den lokalen Akteuren erwähnt und speist sich aus einer sozial erlebten Wirklichkeit, welche diese Akteure neuerdings wieder gerne als Leitmotiv für ihr alltägliches Handeln bzw. ihre politische Haltung angeben. Dieses lässt sich anhand der Studie von Đorđe Tomić (2016) über die Vojvodina zeigen, die eine aufschlussreiche Darstellung von gelebter Raumerfahrung aufweist. Seit den 1980er-Jahren verblasste der Bezug zum sozialistischen Idealstaat Jugoslawien. In diesem Zusammenhang begannen sowohl politische Eliten als auch ,normale' Bürger, sich auf ein Idealbild der Vojvodina als „zivilisiertes, wirtschaftlich wohlhabendes Vielvölkerland in der Mitte Europas“ zu beziehen, um sich vom Rest Serbiens zu unterscheiden und abzugrenzen (ebd., S. 15). Mithilfe des historischen Argumentes seiner früheren politischen Zugehörigkeit zu dem verschwundenen imperialen Reich wurde die Vojvodina „als multikulturell und multikonfessionell gedacht und gleichzeitig als im Wesentlichen unterlegene Gegenposition zum serbischen Nationalismus“ entworfen (ebd., S. 15-16). Im Kontext des post-sozialistischen Umbruchs wird in der neuen Ordnung das ,Alte ${ }^{6}$ als ,authentischer', ,ursprünglicher ${ }^{\star}$ und ,richtiger' wahrgenommen als die vorangegangene Erscheinungsform. Dies kann im Sinne Kosellecks als Umwandlung der Erfahrung, die von den lokalen Akteuren erlebt wird, verstan- 
den werden: Es handelt sich hier nicht um eine rein diskursive Erfindung, sondern um eine verflochtene und selektive Rekonfigurierung des materiellen und immateriellen, sozialen und kulturellen Erbes. Aus dieser Umdeutung der regionalen Erfahrung hat der Phantomraum des habsburgischen Reichs an ,Wirklichkeit' gewonnen. Sie wird von den Akteuren internalisiert und die Phantomgrenze zum ,Balkan' und ,osmanischen Erbe' aufgerufen.

Das Beispiel der Vojvodina weist aber auch auf eine allgemeinere Schlussfolgerung hin: Als Raumerfahrung können Phantomgrenzen und -räume nicht als in sich stabile oder konstante Gegebenheiten verstanden werden; sie werden immer wieder neu bemessen und definiert. In diesem Prozess spielt die zweite Ebene des Phantomgrenzen-Ansatzes, das Raumimaginativ, eine wichtige Rolle.

\subsection{Raumimaginativ}

Auf der Ebene des Raumimaginativs werden Phantomgrenzen in Diskursen, Narrativen sowie mental maps wahrgenommen und tradiert. Sie können als symbolische Grenzen wirken und Bestandteile der mentalen Konstruktionsprozesse geografischer Imaginative sein, welche die Distanz und Differenz zwischen dem, was sie umgrenzen und dem, was sie ausschließen, schaffen und „dramatisieren“ (Said 1978, S. 55). Wiederholt wurden historische Grenzen im Laufe der Geschichte instrumentalisiert, um z.B. die Zivilisation von der Barbarei zu trennen, die Moderne von der Rückständigkeit oder den Reichtum von der Armut. Nationale Großerzählungen haben systematisch aus dem immer vorhandenen großen Repertoire vergangener Grenzen geschöpft, um Identität zu konstruieren, neue Grenzverläufe zu legitimieren bzw. zu entkräften oder um Überlegenheit zu postulieren.

\section{Raumimaginativ am Beispiel des Flüsschen Brynica}

Als Beispiel dient uns hier die Brynica, ein knapp 55 Kilometer langer kleiner Fluss in Südpolen, welcher seit dem 15. Jahrhundert als Scheidelinie zwischen Schlesien und Kleinpolen die Außengrenze des Heiligen Römischen Reiches (und dann ab dem Ende des 19. Jahrhunderts bis 1922 des Deutschen Reiches) bildete. Die historische Arbeit von Jawad Daheur (2017) zeigt anhand von Archivquellen, dass dieses Flüsschen mit der Intensivierung der Staats- und Nationenbildung Deutschlands „eine nationalsymbolische Aufladung“ erfuhr (ebd., S. 158): In seiner Grenzfunktion wurde die Brynica als radikale Scheidelinie zwischen den Kulturen und Symbol für die Abgrenzung der Identitäten in dieser östlichen Grenzregion konstruiert. Trotz seiner geografisch-physischen Bedeutungslosigkeit galt der Fluss bzw. das Gewässer dann nicht nur als imperiale Grenze, sondern als „natürliche“ Grenzscheide „zwischen Schlesien, als Bollwerk der europäischen Kultur und Zivilisation, und Kleinpolen als Vorort der ,asiatischen Barbarei““ (ebd., S. 159). Wie Daheur (ebd.) eindrücklich zeigt, galt die Brynica auch nach 1922 und der Verschiebung der deutsch-polnischen Grenze nach Westen weiterhin als zivilisatorische Grenze im Raumimaginativ der Oberschlesier.

Geografische Imaginative sind nicht nur ein Produkt der herrschenden (Wissens-)Eliten und ihrer Kompetenz und Macht, zum Beispiel ost- oder südosteuropäische Peripherien zu definieren. Sie gehen auch auf das Vermögen der regionalen Akteure zurück, eine räumliche Ordnung Europas ,von unten' mit zu erschaffen. Phantomgrenzen sind nicht ausschließlich politische oder intellektuelle Erfindungen, vorsätzlich geschaffen zu ideologischen Zwecken, 
um Identitäts- oder Hegemoniekonstrukte zu bedienen. Das Konzept der Phantomgrenzen entwickelt seine heuristische Besonderheit, wenn man es auch im sprachlichen Gebrauch und in den impliziten Verwendungen durch lokale Akteure erwägt. Die Narrative regionaler und lokaler Besonderheiten sind in Repräsentationen, in der Sprache (vgl. Schlottmann 2005) oder in Stereotypen verankert und werden (oft unreflektiert) weitergegeben. Aus dieser Perspektive erscheinen die geografischen Imaginationen mit kollektiven und individuellen Erfahrungen verflochten. Die Phantomgrenzen werden ohne ideologische Aufladung in die Dichte der Praktiken integriert. Im Unterschied zu Arbeiten zum mental mapping, welche sich insbesondere auf die Analyse von hegemonialen Diskursen konzentrieren (vgl. zu diesem Thema: Conrad 2002; Schenk 2002), werden die Produktion und Reproduktion geografischer Imaginationen mit dem Phantomgrenzen-Ansatz als in vollem Umfang soziale bzw. gesellschaftliche Vorgänge begriffen, welche in spezifischer Weise auf verschiedenen Ebenen stattfinden können (siehe auch Lehner in diesem Band).

Das Beispiel der Brynica ist auch dafür relevant. Bis zum heutigen Tag wird dieser ehemalige Grenzfluss noch stets als eine ethnisch-kulturelle Grenzlinie imaginiert. Die Erfindung der Brynica als Scheidelinie der Zivilisationen wird heutzutage weiter übermittelt. Daheur (2017) verweist in seiner Arbeit zur Brynica als Instrument der Ost-West-Gliederung auf umfangreiche qualitative sozialwissenschaftliche Forschung, in deren Rahmen auch Interviews mit der Bevölkerung in Städten der Brynica durchgeführt wurden, u.a. in Katowice und Sosnowiec. Diese Studien dokumentieren deutlich, „wie historisch bestimmte Grenzvorstellungen sich in den Raum- und Identitätskonstruktionen der Einwohner widerspiegel[n] “. Die Partikularismen und lokalen Dialekte werden in den Alltagskulturen teilweise weitergepflegt. Bestimmte Stereotype bestehen: Die Oberschlesier werden als „fleißig, stark kirchlich geprägt und Warschau feindlich“ dargestellt. Nach wie vor werden sie mit dem abschätzigen Ausdruck „Hanysy“ (nach dem deutschen Vornamen „Hans“) bezeichnet. Auf der östlichen Seite des Flüsschens werden die Einwohner dagegen für „atheistisch, kommunistisch und Warschau treu eingestellt“ gehalten und „Gorole“ (eigentliche „Bergbewohner“) genannt. Vor Ort tauchen diese Vokabeln in der Alltagssprache sowie in der materiellen Kultur auf und gehören zur lokalen Folklore (ebd., S. 167-171).

So können Gemeinschaften wie regionale, sprachliche oder religiöse Minderheiten Bilder und Symbole von Phantomgrenzen nutzen, um sich im Raum zu verorten und ihrer Erfahrung, ihrer Situation, ihrer Praxis oder ihren Anerkennungsansprüchen Sinn und Folgerichtigkeit zu verleihen. Da sie in ihrer Bindung an natürlich erscheinende Räumlichkeit und Historizität leicht als selbstverständlich erscheinen und diese Selbstverständlichkeit von vielfältigen Narrativen gespeist werden kann, ermöglichen diese ,vertrauten' Phantomgrenzen die Verortung von Identitäten, indem sie den Raum durch Grenzen, die als ,natürlich` wahrgenommen werden, gliedern. Mit anderen Worten: Sie werden nicht nur diskursiv oktroyiert, sondern auch in Praktiken ,von unten' (re-)produziert. Eine solche geografische Vorstellung durchdringt den alltäglichen Sprachgebrauch außerhalb jedweder diskursiven Absicht und trägt dazu bei, eine als selbstverständlich erlebte geografische Wirklichkeit zu erschaffen.

Wichtig ist an dieser Stelle zu betonen, dass solche Phantomgrenzen wandelbar sind. In den geografischen Imaginativen verhält es sich mit den Phantomgrenzen wie mit vermeintlich natürlichen Grenzen, die durch die klassische Geografie festgelegt wurden, um die Welt zu ordnen. Der oben erwähnte symbolische Wert der Brynica in Südpolen zum Beispiel war im 19. Jahrhundert radikal neu, obwohl sie als politische Grenze längst existierte. Erst im 
postsozialistischen Kontext kam es zur Wiederentdeckung dieser Grenze in den mental maps der schlesischen Autonomisten. Neue Hoffnungen, Enttäuschungen oder Erwartungen prägen die Erfahrungen von Akteuren rückwirkend. Die temporale Struktur von Erfahrung schließt also immer auch eine rückwärts wirkende Erwartung ein. Die sich wandelnde Erinnerung an die sozialistische Erfahrung ist ein gutes Beispiel hierfür: War sie Anfang der 1990er-Jahre im Zuge der Transformation von der Plan- zur Marktwirtschaft geprägt von Erinnerungen an Warenknappheit und Warteschlangen, so hat sich diese Erinnerung in den letzten 20 Jahren durch die spätere Erfahrung von Arbeitslosigkeit tiefgreifend verändert: Nun ist es häufig die Erinnerung an Sicherheit und die Vorhersehbarkeit beruflicher Wege, die für viele der ,Verlierer' des postsozialistischen Wandels überwiegt. Dieses Beispiel verdeutlicht, wie gesellschaftliche Erfahrungen und räumliche Bezüge des Alltags aufeinander wirken. Sie können sich über die Zeit ,sinnhaft' reproduzieren und unmerklichem Wandel unterliegen, sich aber auch nach historischen Brüchen innerhalb kürzester Zeit verändern.

\subsection{Raumgestaltung}

Phantomgrenzen werden nicht nur imaginiert und erfahren, sondern gestalten ihrerseits aktiv den Raum mit. Mit dieser dritten Dimension des Phantomgrenzen-Konzeptes wird auf die konkrete Produktion des Raumes hingewiesen, welche sich auf einer anderen analytischen Ebene abspielt als die Produktion von Sinn und die Praktiken der Akteure.

\section{Raumgestaltung an Phantomgrenzen in der Ukraine und in Rumänien}

Anhand einer Studie der Geografin Sabine von Löwis (2014a) kann das Phänomen der Raumgestaltung besonders deutlich gemacht werden. Dabei handelt es sich um die Aufteilung eines vormals einheitlichen Raumes in der Westukraine, in dem heute zwei Dörfer auf den beiden Ufern des Flusses Zbruč koexistieren. Dieser kleine Fluss bildete bis 1918 die Grenze des österreichisch-ungarischen und des Russischen Reiches bzw. zwischen den beiden Weltkriegen zwischen Polen und der Sowjetunion. In ihrer Studie untersucht Löwis in den beiden ,Zwillingsdörfern` Räume der Identifikation und der politischen Orientierung der Bewohner. Sie betrachtet sowohl die Denkmalkultur als auch das Selbstverständnis der Einwohner auf der jeweiligen Seite des Zbruč. Dabei stellt sich heraus, dass weder die in den Denkmälern berücksichtigten Ereignisse noch die dort gefeierten Personen identisch sind, trotz der physischen Nähe und dem identischen Ursprung des Städtchens: Im Dorf auf der Ostseite des Flusses erinnern die Denkmäler an kommunistische Zeiten, die jeweiligen Anführer wie auch die durchgeführten Kriminalitätsakte, dagegen wird im Dorf westlich der alten Grenze dem ukrainischen, antibolschewistischen Nationalismus gedacht.

Die Zeugnisse der befragten Einwohner weisen dagegen keine expliziten Gegensätze bezüglich ihrer Historie und Struktur auf. Der Bezug auf die Monumente ist eher instabil. Somit zeigt Löwis, dass es keine mechanistische intragenerationelle Weitergabe politischer Traditionen oder sozialer Normen gibt, z.B. das die Einwohner im westlichen Dorf ,pro-ukrainisch“ und die im östlichen Teil ,pro-russisch' wären. Vielmehr erscheint die - von oben oktroyierte - Wahl bestimmter Erinnerungsorte als Motor, welcher wiederum durch die Notwendigkeit induziert wurde, dem jungen ukrainischen Staat eine nationale Identität zu geben: Die Bewohner des östlichen Dorfs identifizierten sich dabei eher mit der Erinnerung an die große Hungersnot 
(Olodomor) von 1935 und mit Erfahrungen an die Sowjetunion; die Bewohner des westlichen Dorfes konnten (natürlich) keine Episode hervorheben, an der sie nicht selber teilgenommen hatten, und hielten daher die Armee des ukrainischen Aufstands hoch. Die beiden Dorfgemeinschaften schaffen somit zwei deutlich unterschiedliche Erzählungen auf der Grundlage unterschiedlicher Interpretationen des großen Nationalepos, welches die Zentralmacht auf die Tagesordnung gesetzt hatte (ebd., S. 158-160).

Anhand des Beispiels lässt sich erkennen, wie der Fokus auf die Akteure als Handelnde die Raumgestaltung zu einem alltäglichen Prozess macht: Die Subjekte gestalten ihre Räume nicht zufällig, beliebig, aus freien Stücken, sondern auf der Basis eines vorgegebenen, aber ständig neu interpretierten Raumverständnisses. Der in der longue durée (vgl. Braudel 1984) entstandene, vorgegebene Raum wird dabei nicht als determinierend für die Handlung der Akteure verstanden, sondern mit den konkreten und manchmal eigensinnig erscheinenden Strategien der Akteure ins Verhältnis gesetzt. Fortschreibung oder Wandel von vorgegebener Räumlichkeit sind immer kontextgebunden. Aus solcher Perspektive, von unten` lassen sich die strukturellen sowie die diskursiven Prägungen des Raumes in ihrer performativen Wirksamkeit - gleichsam als soziale Praxis - betrachten (Esch/Hirschhausen 2017, S. 15).

Die hier beschriebenen raumgestaltenden Effekte beschränken sich nicht auf die Aufteilung der Räume im Beispiel der Phantomgrenzen in der Ukraine, sie haben darüber hinaus auch für die ,normale‘ Raumgestaltung im Alltagsleben Bedeutung. Dies kann anhand einer banal erscheinenden Infrastruktur des täglichen Gebrauchs beispielhaft aufgezeigt werden: Die vergleichende Studie der Modernisierung der Wasserinfrastruktur in ländlichen Gegenden Rumäniens (vgl. Hirschhausen 2015; 2017) fragt danach, warum sich nach dem Jahr 2000 die ländlichen Modernisierungsstrategien so stark regional unterschieden. Sie waren in den Gegenden stark gegensätzlich, welche durch die alte (Phantom-)Grenze entlang der Karpaten bis 1918 geprägt waren und die Territorien Österreich-Ungarn von den rumänischen Fürstentümern trennte. Seit der Jahrhundertwende rüsten viele Haushalte des Banats und Transsilvaniens, also westlich der genannten Grenze, ihre Häuser wesentlich rascher mit einer fließenden Wasserversorgung aus als ihre Nachbarn östlich dieser Grenze. Dies führt zu einer sukzessiven Sichtbarwerdung des Phänomens in offiziellen Statistiken und Erhebungen der Haushaltssituationen.

Neben allgemeinen statistischen Erhebungen wurden in je zwei Dörfern auf beiden Seiten der Grenze persönliche Fallstudien zu dieser aufkommenden Asymmetrie durchgeführt („Geographie der Wasserinfrastruktur“, Hirschhausen 2015). Dabei ergab sich, dass die Unterschiede nicht durch eine bloße Rekonstruktion einer historisch dauerhaft akzeptierten Superiorität Mitteleuropas gegenüber einem rückständigen Balkan-Europa erklärbar sind. Vielmehr ist die Differenz Ausdruck unterschiedlich aufgefasster Erwartungen bezüglich der vermuteten sozialen und wirtschaftlichen Zukunft und lokaler Möglichkeiten, diese anzugehen. Es handelt sich daher eher um eine vorwärtsschauende Perspektive, bei der die lokalen Akteure sich selbst bzw. ihre Dörfer in die Zukunft projizieren. Die Fallstudie vermittelt auch die Erklärungsstärke der mental maps bei der Raumgestaltung, welche die Imaginative (mittel- und balkan-)europäischer Perspektiven widerspiegeln und dabei Mitteleuropa eine günstige Entwicklung und dem Balkan-Europa ein Zurückbleiben anhängen. Der Ansatz ist umso anschlussfähiger, als dass der Unterschied von den lokalen Akteuren als ,natürlich' empfunden wird und diese nicht aus dem ökonomischen Schema ausbrechen. Die dadurch geschaffenen Räume und (Phantom-)Grenzen werden durch tagtägliche Praktiken aktualisiert und sind gleichsam das Spiegelbild der langfristigen Vision. So gilt auch für die Raumgestaltung, dass die Spuren der 
Vergangenheit (auch) aus der Vision der Zukunft stammen (vgl. hierzu auch Leutloff-Grandits in diesem Band).

Die dargestellten Beispiele zeigen auch, dass sich die drei Ebenen von Raumerfahrung, imaginativ und -gestaltung überlappen und auch gegenseitig bedingen. Sie sind ineinander und miteinander verschränkt, sie wirken zusammen und beeinflussen sich gegenseitig, ohne ineinander vollständig aufzugehen: Insgesamt konstituieren sie die Art, in der soziale Strukturen, Praktiken und Diskurse sich verräumlichen und umgekehrt, wie der soziale Raum in seinen drei Dimensionen Strukturen, Akteure und Diskurse zueinander in Beziehung setzt. Über die Akteure kann man am einfachsten den Zugang zum Zusammenwirken der drei Dimensionen suchen: Soziale Wirklichkeit und sozial konstruierte Raum-Zeit entstehen in ihrer Artikulation zu- und miteinander.

\section{Abschließende Definition von Phantomgrenzen und Fazit}

Bei der abschließenden Definition des Konzepts der Phantomgrenzen (und der durch sie geschaffenen Phantomräume) wird auf das oben Beschriebene Bezug genommen, aber über das klassische Raum-Zeit-Schema hinweggegangen: Zwar interessieren die Weitertradierung oder Wiedererfindung von kulturellen Mustern in der Longue durée bei regionalen Gesellschaften, jedoch verstehen wir diese weder als akkumuliertes Sozialkapital (social capital, vgl. Putnam 1994; Becker et al. 2016) noch als cultural legacies (Peisakhin 2016) oder pfadabhängige Prozesse (path dependent, vgl. Pierson 2004). Das Konzept Phantomräume bzw. Phantomgrenzen grenzt sich von diesen klassischen Perspektiven durch drei Merkmale ab:

- Das Konzept der Phantomräume hält die Akteure nicht in historisch etablierten, regionalen Strukturen ,gefangen', vielmehr berücksichtigt es die Autonomie der Akteure. Dabei werden regionalspezifische Anordnungen durch das alltägliche Handeln produziert, aktualisiert oder abgeschafft. Historisch vererbte Praktiken oder Imaginative werden nicht mechanisch tradiert, sondern selektiv erinnert bzw. vergessen, aktualisiert oder disqualifiziert.

- Die durch zahlreiche Handlungen von Akteuren hervorgerufenen Veränderungen regionaler Anordnungen lassen sich im Verlauf der Zeit aus sich ändernden Zuordnungen von Erfahrungen und Erwartungen ableiten. Dadurch wird die Historizität dieser räumlichen Gebilde geprägt. Der „Erfahrungsraum“ wird durch den „Erwartungshorizont“ (Koselleck 1979) von den Akteuren ständig neu ausgewertet. Dies führt dazu, dass die Transformation bisher erfahrener Kulturräume auch aus der Perspektive imaginierter Zukunft erfolgt: Die Akteure schaffen regionale Anordnungen nicht nur aus ihren „wirklichkeitsgesättigten“ Erfahrungen (ebd., S. 357) sondern auch aus ihren imaginationsgeprägten Erwartungen.

- Imaginierte Zukunft entsteht dabei nicht nur endogen, innerhalb lokaler oder regionaler Erfahrungen. Geprägt wird sie auch durch imaginierte Geografien auf einer ganz anderen Ebene hegemonialen Wissens, welches außerhalb der Region produziert wird. Solche mental maps zeigen Zentren und Peripherien, modernere und rückständigere Regionen und behaupten dadurch eine Geografie der Zukunft, die von großen Narrativen sowie von Prophezeiungen aufgeladen sind. Auf der Akteursebene erhalten sie ihre Kraft aus ihrer wahrgenommenen, Natürlichkeit‘.

Zusammenfassend lässt sich festhalten, dass Phantomgrenzen (und Phantomräume) die performative Wirkung ehemaliger historischer Territorien bezeichnen. Frühere historische Raumordnungen können die Erfahrung sowie das Raumimaginativ von sozialen Gruppen prägen und 
daher bestimmte regionale Ordnungen neu schaffen (perform). Diese Fähigkeit ist instabil, aber historisch situiert. Phantomgrenzen und -räume können in bestimmten historischen sowie geografischen Kontexten erscheinen oder verschwinden. In diesem Sinne bietet das Konzept der Phantomgrenzen einen neuen oder alternativen Zugang zur Regionalforschung, welcher gerade in anderen als den hier erforschten Räumen Ostmitteleuropas neue Perspektiven erschließen kann. Zukünftige Forschung sollte auch andere Regionen mit ,Zwischenräumen` und kolonialem Erbe adressieren, wie z.B. den Kaukasus, Nordafrika und den mittleren Osten (MENA-Region) sowie Subsahara-Afrika.

\section{Weiterführende Literatur}

Aldenhoff-Hübinger, Rita/Goussef, Catherine/Serrier, Thomas (Hrsg.) (2007): Europa Vertikal. Zur OstWest-Gliederung im 19. und 20. Jahrhundert. Göttingen: Wallstein Verlag.

Esch, Michael/Hirschhausen, Béatrice von (2017): Einleitung. In: Dies. (Hrsg.): Wahrnehmen, Erfahren, Gestalten. Phantomgrenzen und soziale Raumproduktion. Göttingen: Wallstein Verlag, S. 7-48.

Hirschhausen, Béatrice von/Grandits, Hannes/Kraft, Claudia/Müller, Dietmar/Serrier, Thomas (2019): Phantom Borders in Eastern Europe. A New Concept for Regional Research. In: Slavic Review 78, H. 2, S. 368-389.

Hirschhausen, Béatrice von/Grandits, Hannes/Kraft, Claudia/Müller, Dietmar/Serrier, Thomas (2015): Phantomgrenzen: Räume und Akteure in der Zeit neu denken. Göttingen: Wallstein Verlag.

Löwis, Sabine von (Hrsg.) (2015): Phantom Borders in the Political Geography of East Central Europe. In: ERDKUNDE 69, H. 2, S. 99-186.

\section{Literaturverzeichnis}

Ash, Timothy Garton (1986): Does Central Europe exist? In: The New York Review of Books 33, H. 15, S. 45-52.

Becker, Sascha O./Boeckh, Katrin/Hainz, Christa/Woessmann, Ludger (2016): The Empire is dead, long live the empire! Long-run persistence of trust and corruption in the bureaucracy. In: The Economic Journal 126, H. 590, S. 40-74.

Barth, Fredrik (1969/1998). Ethnic Groups and Boundaries: The Social Organization of Cultural Difference. Long Grove: Waveland Press.

Braudel, Fernand (1984). Geschichte und Sozialwissenschaften - Die „longue durée“. In: Wehler, Hans-Ulrich (Hrsg.): Geschichte und Soziologie. Königstein/Taunus: Athenäum, S. 189-215.

Conrad, Christoph (Hrsg.) (2002): Mental Maps. In: Geschichte und Gesellschaft 28, H. 3, S. 337-514.

Daheur, Jawad (2017): Die Brynica als Instrument der Ost-West-Gliederung: Zur Karriere eines Grenzflüsschens in der langen Dauer. In: Aldenhoff-Hübinger, Rita/Goussef, Catherine/Serrier, Thomas (Hrsg.): Europa Vertikal. Zur Ost-West-Gliederung im 19. und 20. Jahrhundert. Göttingen: Wallstein Verlag, S. 155-172.

Esch, Michael/Hirschhausen, Béatrice von (2017): Einleitung. In: Dies. (Hrsg.): Wahrnehmen, Erfahren, Gestalten. Phantomgrenzen und soziale Raumproduktion. Göttingen: Wallstein Verlag, S. 7-48.

Foucher, Michel (1993): Fragments d'Europe. Atlas de l'Europe médiane et orientale. Paris: Fayard.

Grandits, Hannes/Hirschhausen, Béatrice von/Kraft, Claudia/Müller, Dietmar/Serrier, Thomas (2015): Phantomgrenzen im östlichen Europa. Eine wissenschaftliche Positionierung. In: Dies. (Hrsg.): Phantomgrenzen: Räume und Akteure in der Zeit neu denken. Göttingen: Wallstein Verlag, S. 13-56.

Gregory, Derek (1994): Geographical Imaginations. Malden: Wiley-Blackwell.

Grosfeld, Irena/Zhuravskaya, Ekaterina (2014): Persistent effects of empires: Evidence from the partitions of Poland. In: Paris School of Economics, Working Paper Nr. 05. www.halshs.archives-ouvertes.fr/halsh s-00795231/document, 25.09.2018.

Hartshorne, Richard (1933): Geographic and Political Boundaries in Upper Silesia. In: Annals of the Association of American Geographers 23, H. 4, S. 195-228. DOI: 10.1080/00045603309357073.

Hirschhausen, Béatrice von (2017): The heuristic interest of the concept of "phantom borders“ in the understanding of cultural regionalization. In: L'Espace géographique 46, H. 2. www.cairn-int.info/journ al-espace-geographique-2017-2.htm, 25.09.2018.

Hirschhausen, Béatrice von/Grandits, Hannes/Kraft, Claudia/Müller, Dietmar/Serrier, Thomas (2015): Phantomgrenzen: Räume und Akteure in der Zeit neu denken. Göttingen: Wallstein Verlag.

Hirschhausen, Béatrice von/Grandits, Hannes/Kraft, Claudia/Müller, Dietmar/Serrier, Thomas (Jahreszahl): „Phantom Borders in Eastern Europe. A New Concept for Regional Research“. In: Slavic Review 78, H. 2, S. 368-389. 
Koselleck, Reinhart (1979): Vergangene Zukunft. Zur Semantik geschichtlicher Zeiten. Frankfurt/M.: Suhrkamp Taschenbuch.

Kraft, Claudia (2002): Europa im Blick der polnischen Juristen. Rechtsordnung und juristische Profession in Polen im Spannungsfeld zwischen Nation und Europa 1918-1939. Frankfurt/M.: Vittorio Klostermann Verlag.

Kundera, Milan (1983): Un occident kidnappé ou La tragédie de l'Europe centrale. In: Le Débat 25, H. 5, S. 3-23.

Kundera, Milan (1987): Un occident kidnappé oder die Tragödie Zentraleuropas. www.europa.clio-online .de/quelle/id/artikel-3287, 25.09.2018.

Lefebvre, Henri (1974): La production de l'espace. Paris: éditions Anthropos.

Löwis, Sabine von (2014a): Ambivalente Identifikationsräume in der Westukraine. Das Phantom der alten Grenze am Zbruč. In: Europa Regional 22, H. 3/4, S. 148-162.

Löwis, Sabine von (Hrsg.) (2014b): Einführung - zur Zeitlichkeit räumlicher Konstrukte: Grenzen und Regionen in Vergangenheit und Gegenwart. In: Europa Regional 22, H. 3/4, S. 83-89.

Löwis, Sabine von (Hrsg.) (2015): Phantom Borders in the Political Geography of East Central Europe. In: ERDKUNDE 69, H. 2, S. 99-186.

Müller, Michael G./Struve, Kai (Hrsg.) (2017): Fragmentierte Republik? Das politische Erbe der Teilungszeit in Polen 1918-1939. Göttingen: Wallstein Verlag.

Müller, Uwe (2014). Instrumente imperialer Politik? Eisenbahnen in Ostmitteleuropa im 19. Jahrhundert. In: Mitropa. Jahresheft des Geisteswissenschaftlichen Zentrums Geschichte und Kultur Ostmitteleuropas 2014, S. 20-24.

Peisakhin, Leonid (2015): Cultural Legacies: Persistence and Transmission. In: Norman, Schofield/Gonzalo Caballero (Hrsg.): The Political Economy of Governance Institutions, Political Performance and Elections. Cham: Springer International Publishing, S. 21-39.

Pierson, Paul (2004): Politics in Time: History, Institutions, and Social Analysis. Princeton/Chichester: Princeton University Press.

Puttkamer, Joachim von (2010): Ostmitteleuropa im 19. und 20. Jahrhundert. Oldenbourg Grundriss der Geschichte, Bd. 38. München: Oldenbourg.

Putnam, Robert (1994): Making democracy work: Civic traditions in modern Italy. Princeton/Chichester: Princeton University Press.

Roubitschek, Walter (2004): Die ostdeutsche Landwirtschaft: Umbruch und Erneuerung. In: Haas, HansDieter (Hrsg.): Unternehmen und Märkte, National Atlas, Bd. 8. Heidelberg: Spektrum Akademischer Verlag, S. 118-121.

www.archiv.nationalatlas.de/wp-content/art_pdf/Band8_118-121_archiv.pdf, 25.09.2018.

Said, Edward W. (1978): Orientalism. London: Routledge \& Kegan Paul Ltd.

Schenk, Benjamin F. (2002): Mental Maps. Die Konstruktion von geographischen Räumen in Europa seit der Aufklärung. Literaturbericht. In: Geschichte und Gesellschaft, Bd. 28, Sp. 493-514.

Schlottmann, Antje (2005): RaumSprache. Ost-West-Differenzen in der Berichterstattung zur deutschen Einheit. Eine sozialgeographische Theorie. Stuttgart: Franz Steiner Verlag.

Siegrist, Hannes/ Müller, Dietmar (Hrsg.) (2015): Property in East Central Europe: Notions, Institutions and Practices of Landownership in the Twentieth Century. New York / Oxford: Berghahn.

Sundhaussen, Holm (1999): Europa balcanica. Der Balkan als historischer Raum Europas. In: Geschichte und Gesellschaft, Bd. 25, Nr. 4, S. 626-653.

Sundhaussen, Holm (2003): Der Balkan: Ein Plädoyer für Differenz. In: Geschichte und Gesellschaft 29, H. 4, S. 608-624.

www.cairn-int.info/journal-espace-geographique-2017-2.htm, 25.09.2018.

Ther, Philippe (2003). Einleitung. In: Ders./Sundhaussen, Holm (Hrsg.): Regionale Bewegungen und Regionalismen in europäischen Zwischenräumen seit der Mitte der 19. Jhd. im Vergleich. Marburg: Herder-Institut Verlag.

Todorova, Maria (1997): Imagining the Balkans. New York: Oxford University Press.

Todorova, Maria (2002): Der Balkan als Analysekategorie: Grenzen, Raum, Zeit. In: Geschichte und Gesellschaft 28, H. 3, S. 470-492.

Tomić, Đorđe (2016): Phantomgrenzen und regionale Autonomie im postsozialistischen Südosteuropa. Die Vojvodina und das Banat im Vergleich. Göttingen: Wallstein Verlag.

Troebst, Stefan (2010): „Geschichtsregion“: Historisch-mesoregionale Konzeptionen in den Kulturwissenschaften. In: Europäische Geschichte Online (EGO), Institut für Europäische Geschichte (IEG), Mainz. www.ieg-ego.eu/troebsts-2010-de, 22.08.2020.

Zarycki, Tomasz (2015): The electoral geography of Poland: Between stable spatial structures and their changing interpretations. In: ERDKUNDE 69, H. 2, S. 125-137. 